In der Rubrik „Literatur kompakt" werden die wichtigsten Originalarbeiten aus der internationalen Fachliteratur referiert.

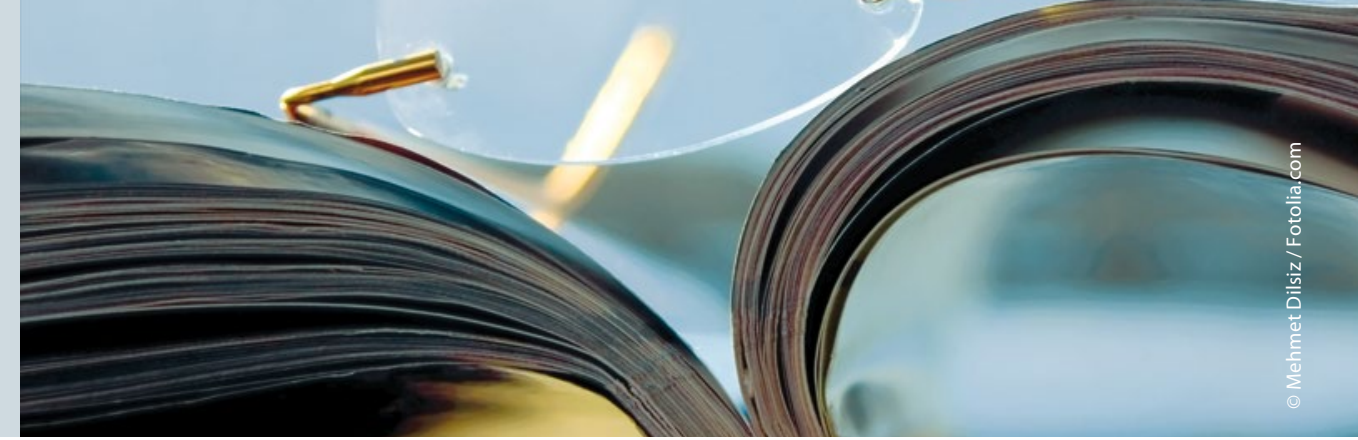

\section{„Pille danach“: Mit der Rezeptfreiheit steigt die Nachfrage}

Inzwischen können Frauen, die eine Notfallkontrazeption wünschen, die "Pille danach" rezeptfrei in der Apotheke erhalten. Ob und wie sich mit dem leichteren Zugang das Nutzungsverhalten geändert hat, wurde aktuell von einem Team um Prof. Marion Kiechle untersucht.

Z um 15. März 2015 wurden die Wirkstoffe Levonorgestrel (LNG) und Ulipristalacetat (UPA) zur Notfallkontrazeption von der Rezeptpflicht befreit. Eine Entscheidung, die umstritten ist. Die orale Notfallkontrazeption muss möglichst zeitnah - am besten innerhalb der ersten 24 Stunden nach ungeschütztem Verkehr eingenommen werden - denn mit jeder Verzögerung sinkt die Effektivität der Methode.

Wie sich die Situation nun ein Jahr nach der OTC-Freigabe darstellt, hat ein Team um Prof. Marion Kiechle, Frauenklinik der Technischen Universität,
München, untersucht. Laut Zahlen der IMS HEALTH stieg mit der Entbindung von der Rezeptpflicht die Menge der abgegebenen Packungen von 40.000 auf 52.000 pro Monat. 3,9\% der Frauen zwischen 15 und 49 Jahren hatten 2015 die "Pille danach" eingenommen. Damit liegt der Anteil hierzulande immer noch unter dem europäischen Durchschnitt $(6,7 \%)$ und weit hinter den Spitzenreitern Schweden und Frankreich mit $11,5 \%$ bzw. $11,4 \%$. Mehr als zwei Drittel der Nutzerinnen waren über 20 Jahre und die meisten gaben als Grund für die orale Notfallkontrazeption an, dass das

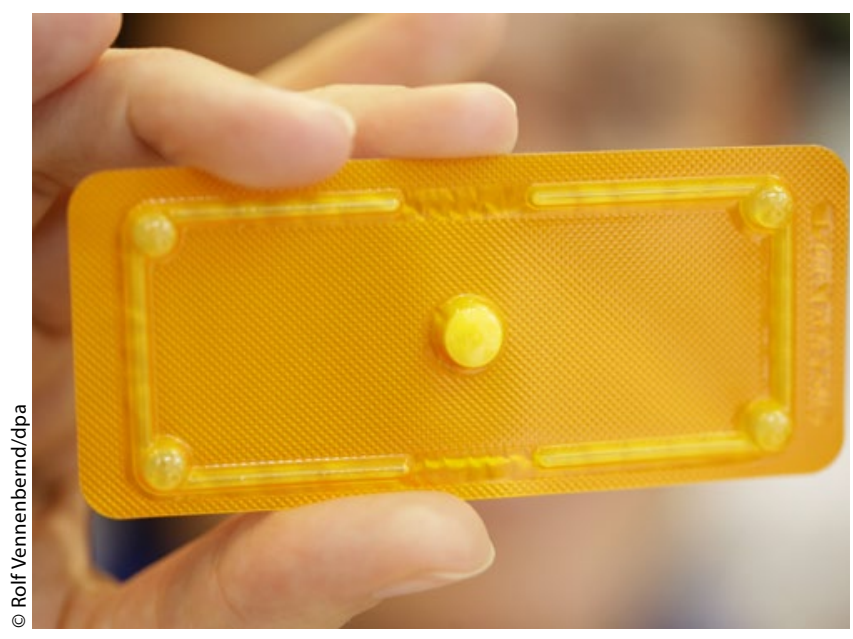

Vor knapp zwei Jahren wurde die „Pille danach" aus der Verschreibungspflicht entlassen. Das Nutzungsverhalten in Deutschland hat sich dadurch verändert.
Kondom gerissen sei (39\%) oder die Pilleneinnahme vergessen wurde (34\%). Erwartungsgemäß ist die Nachfrage vor allem am Wochenende gestiegen.

Auf die Zahl der Schwangerschaftsabbrüche hatte der OTC-Status keinen Einfluss. Wurden 2014 insgesamt 99.715 Schwangerschaften abgebrochen, waren es 99.237 Schwangerschaften 2015. Es fiel jedoch auf, dass in Bundesländern, in denen der Anteil der „Pille-danach“Nutzerinnen höher lag, weniger Schwangerschaften abgebrochen wurden als in Bundesländern mit niedrigerem Anteil.

Das Alter der Anwenderinnen sank, anders als befürchtet, mit dem OTC-Status nicht, wie die Autoren betonen. Infolge des leichteren Zugangs verringerte sich im Durchschnitt die Zeitspanne zwischen ungeschütztem Verkehr und Pilleneinnahme, was angesichts des Wirkmechanismus eine wichtige Entwicklung sei.

Die Daten zeigen aber auch, dass allein mit dem rezeptfreien Erwerb die Zahl der Schwangerschaftsabbrüche nicht gesenkt werden könne. Dafür seien die Zusammenhänge $\mathrm{zu}$ komplex, erklären Kiechle et al. Ein wichtiger Grund könnte der nach wie vor geringe Kenntnisstand in der Bevölkerung sein. Beispielweise wisse nur etwa die Hälfte der 16-39-Jährigen überhaupt von der Möglichkeit einer oralen Notfallkontrazeption, und 94\% der Frauen, die 2015 ungewollt schwanger wurden und abgetrieben haben, hätten nach ungeschütztem Verkehr keinerlei Versuche gestartet, eine Schwangerschaft zu verhindern.

Dr. Dagmar Kraus

Kiechle $M$ et al. Experience with oral emergency contraception since the OTC switch in Germany. Arch Gynecol Obstet 2016, online 28. November 